\title{
Atomic-Scale Analysis of Chemical Bonding of Delaminated Graphene at Faceted SiC by Aberration-Corrected Scanning Transmission Electron Microscopy
}

\author{
G Nicotra $^{1}$, Q M Ramasse $^{2}$, I Deretzis ${ }^{1}$, C Bongiorno $^{1}$, C Spinella $^{1}$ and F Giannazzo ${ }^{1}$ \\ 1. Istituto per la Microelettronica e Microsistemi (CNR-IMM), VIII strada 5, I-95121 Catania, Italy \\ 2. SuperSTEM Laboratory, STFC Daresbury, Keckwick Lane, Daresbury WA4 4AD, UK
}

Graphene is a single-atom-thick crystal of $\mathrm{sp}^{2}$-hybridized carbon atoms that exhibits exceptional electronic properties, which make it a promising material for post-CMOS applications and for ultrafast electronics operating in the $100 \mathrm{GHz}$ to $\mathrm{THz}$ frequencies. Optimal performance in graphene based devices depends on the high mobility and long lifetimes of carriers. Within this framework, among the different routes for the synthesis of large-area graphene sheets, epitaxial growth on $\mathrm{SiC}$ substrates has emerged as one of the principal technologies. Controlled graphitization of hexagonal $\mathrm{SiC}$ by high-temperature thermal processes is the strongest candidate for graphene-based electronics, owing to the fact that few-layers-graphene (FLG) can be grown on a semiconductor substrate without any need for transfer (as is the case with CVD-grown graphene on metal substrates). Graphene produced in such a way is known as epitaxial graphene (EG) [1]. However the substrates that support graphene structures may break the ideal symmetry or dope the graphene with extrinsic charge, either of which profoundly affects the electrical transport [2]. A clear evidence of the interaction between graphene and Si-terminated $\mathrm{SiC}$ surfaces is the reported presence of an interface (buffer) layer [3]. This SiC interfacial structure may play an important role in the transport properties of the graphene grown epitaxially on top of it and it is therefore of fundamental importance to examine the transport properties with atomic-scale measurements in this region.[4]. This represents a challenge for the graphene-SiC approach mainly due to the limited understanding of the interface's influence on the electronic properties and charge transport.

To figure out the role of the substrate in this graphene system, we carried out observations of a stack of 4 graphene layers grown epitaxially on a $4-\mathrm{H} \mathrm{SiC} \mathrm{substrate,} \mathrm{using} \mathrm{a} \mathrm{Nion} \mathrm{UltraSTEM}{ }^{\mathrm{TM}} 100$ dedicated aberration-corrected scanning transmission electron microscopes (STEM). The microscope was operated in so-called 'gentle STEM' conditions [5] at $60 \mathrm{keV}$ primary beam energy, with the probe-forming optics configured to provide a beam convergence half-angle of $30 \mathrm{mrad}$ for an estimated probe size of $1.1 \AA$. The microscope is also equipped with a cold field emission gun with a native energy of $0.35 \mathrm{eV}$ in conditions used here. The combination of very high spatial resolution with the possibility to work at an operating energy that is below the knock-on threshold for carbon [6] was essential for this work. Under these conditions, atoms in the structure are clearly separated in the high angle annular dark field (HAADF) images, while long electron energy loss spectroscopy (EELS) acquisitions are possible without damaging the EG structure. Figure 1a,b show atomic-scale EELS analysis across the interface and atomic resolution HAADF-STEM images in cross-section of the $\mathrm{SiC} /$ Graphene interface, respectively. In the STEM image we can easily identify three graphene layers on the topmost side where individual carbon columns are distinguishable. Moreover, the image clearly reveals the presence of a fourth buffer layer at the interface with the SiC. The atomic-scale EELS analysis shows clear differences in the fine structure of the $\mathrm{C} \mathrm{K}$ edge as the probe is positioned serially on each of the layers. As is well-known, a sharp peak appears at $285 \mathrm{eV}$ when $1 \mathrm{~s}$ to $\pi^{*}$ transitions are present. This peak is clearly visible and sharp on the first and the second layer of graphene: intensity of the spectral signal specific to $\pi$ bonding is associated with the presence of $\mathrm{sp}^{2}$-hybridization as is expected in graphene. The peak intensity drops at the buffer layer, indicative of a reduction of $\pi^{*}$ bonding and a simultaneous increase of $\sigma^{*}$ bonding. These results would suggest the presence of $\mathrm{sp}^{3}$-hybridization (adamantine), possibly responsible for the electron transport degradation.

The combination of atomic resolution HAADF-STEM with high energy resolution EELS allowed us to obtain punctual information on the degree of hybridization $\mathrm{sp}^{2}$ (graphitic nature) of this buffer layer. In fact this technique opened the way to the punctual evaluation of the $\mathrm{sp}^{2}$ fraction of EG at the atomic scale, that will be extended to the wrinkles and terrace kinks edge, as the ones reported in Figure 2, suspected to be responsible for a degradation of the expected electrical behavior. 


\section{References}

[1] C Vecchio et al., Nanoscale Research Letters 6:(2011), 269.

[2] C. Berger, et al., Science 312, 1191 (2006).

[3] J Borysiuk, et al., J. Appl. Phys. 105 (2009), 023503.

[4] G. M. Rutter et al., Science 317, 219 (2007).

[5] O.L. Krivanek et al., Ultramicroscopy 108 (2008), 179.

[6] O.L. Krivanek et al., Nature 464 (2010), 571-574.

[7] The authors gratefully acknowledge Ondrej L. Krivanek and Niklas Dellby from Nion Co. for giving us the opportunity to interface with the SuperSTEM Laboratory, STFC Daresbury.

Figure 1. a) atomic-EELS spectra of the C K-edge collected in the SiC bulk, on the buffer layer, on the $1^{\text {st }}$ and
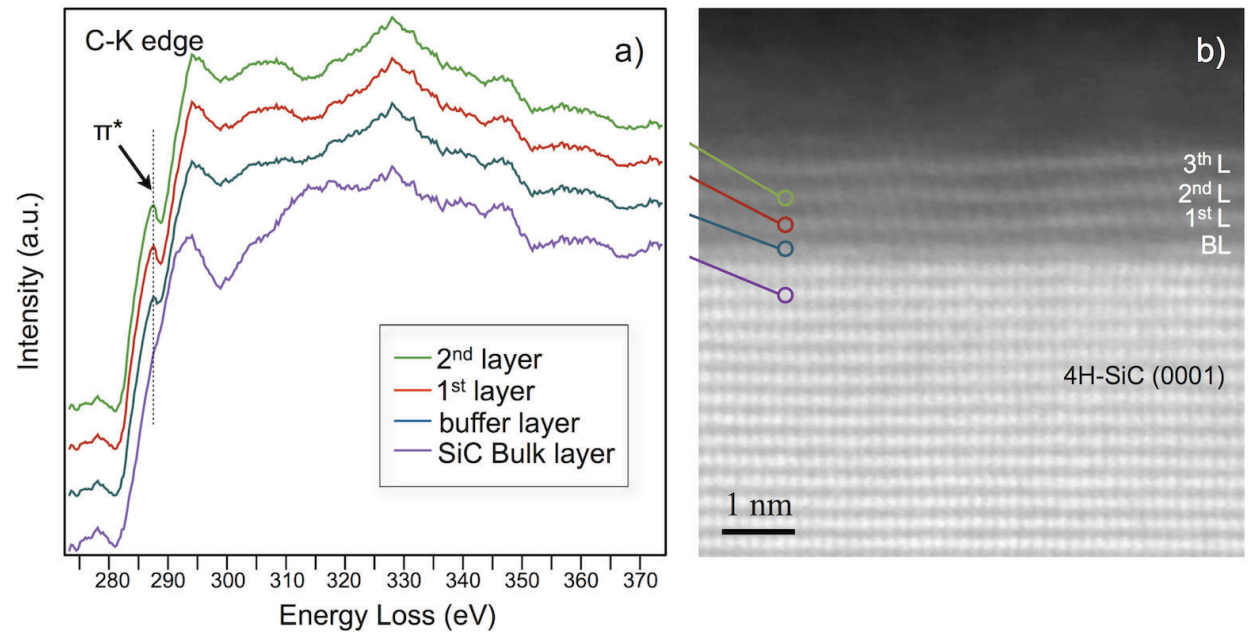

$2^{\text {nd }}$ graphene flat layers of the left image, acquired with an estimated probe size of 1.1A b) 60keV HAADF STEM image of planar 4H-SiC (0001) Si-terminated SiC.Three epitaxial graphene layer are clearly resolved at atomic-scale together with the graphene buffer layer.

Figure 2. 60keV HAADF STEM images, acquired at different magnifications, showing kinks terrace edge on
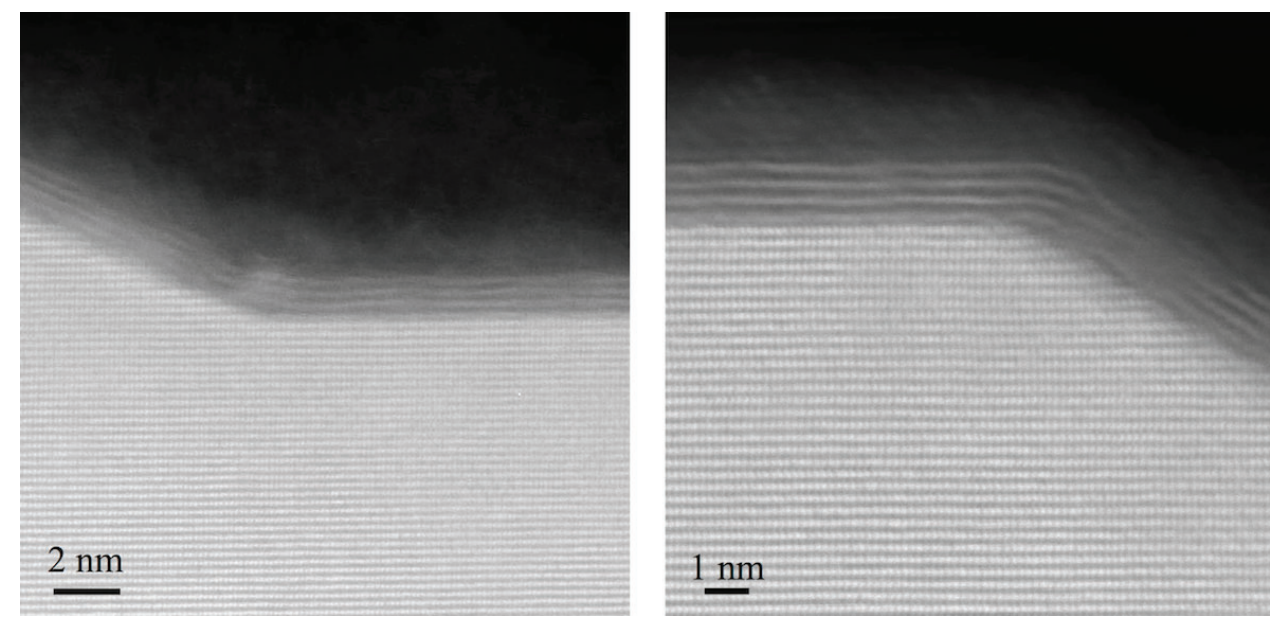

4H-SiC (0001) Si-terminated SiC. Defects along the kink terrace edge are clearly visible and prevent some graphene from forming continuous layers. 